
\title{
25 Research Sourere \\ Inflammatory Bowel Disease and the Risk of Prostate Cancer: A Meta-analysis of Cohort Studies
}

\section{Wen-Qing Lian ( $\nabla 742808925 @ q q . c o m$ )}

Zhongshan Affiliated Hospital of Sun Yat-sen University

Hong-Xing Huang

Zhongshan Affiliated Hospital of Sun Yat-sen University

\section{Fa-Jiang Li}

Zhongshan Affiliated Hospital of Sun Yat-sen University

\section{Liang-Hua Chen}

The Thirs Affiliated Hospital of Southern Medical University

\section{Research article}

Keywords: Inflammatory bowel disease, prostate cancer, meta-analysis, Crohn's disease, ulcerative colitis

Posted Date: August 30th, 2019

DOI: https://doi.org/10.21203/rs.2.13761/v1

License: (c) (i) This work is licensed under a Creative Commons Attribution 4.0 International License. Read Full License 


\section{Abstract}

Purpose This meta-analysis aims to assess the prostate cancer risk in patients with inflammatory bowel disease (IBD).

Methods A systematic search was conducted on PubMed, EMBASE, and the Cochrane Library databases for eligible studies published before April 2019. Pooled relative ratios (RRs) and 95\% confidence intervals (Cls) were estimated to evaluate the relationship between history of IBD and prostate cancer risk.

Results Fourteen publications involving thirteen cohort studies were eligible. The meta-analysis showed that IBD markedly elevated risk of prostate cancer (RR 1.36, 95\% Cl 1.11-1.67). Subgroup analysis by subtype of IBD showed that ulcerative colitis was associated with a statistically significant increase in risk of prostate cancer (RR 1.30, 95\% Cl 1.09-1.55), while Crohn's disease was not (RR 1.09, 95\% Cl 0.901.34).

Conclusion The present meta-analysis indicates that IBD increases the risk of prostate cancer, particularly in ulcerative colitis men. More prospective cohort studies are required to confirm our findings.

\section{Introduction}

Prostate cancer ( $\mathrm{PCa}$ ) is the second most frequently diagnosed malignancy and the fifth leading cause of cancer deaths worldwide. There will be an estimated 1276106 new cases and 358989 newly related deaths assigned to PCa in 2018 [1]. It is important to identify effective methods for preventing or at least early detecting PCa. However, the development of PCa is complex and its exact etiology remains unclear. Several risk factors associated with PCa include age, race, lifestyle, and family history. Epidemiologic researchers also have noted the influence of chronic inflammation on PCa [2-4].

Inflammatory bowel disease (IBD), including Crohn's disease (CD) and ulcerative colitis (UC), is characterized by chronic relapsing inflammatory condition of the gastrointestinal tract [5]. Earlier population-based studies and meta-analyses demonstrate that patients suffering from IBD are at an increased risk of intestinal and extraintestinal cancers [6]. Although there are quite a lot of epidemiologic studies trying to clarify the association between risk of PCa and history of IBD, the conclusions are still inconsistent [7-21]. We thereby performed this meta-analysis to comprehensively assess the association between them.

\section{Methods}

\section{Search strategy}

Our meta-analysis was conducted according to the PRISMA guidelines (Additional file 1: Data S1) [22] and MOOSE guidelines [23]. We searched in PubMed, EMBASE, and Cochrane Library databases to identify potentially eligible papers published before April 2019. The search terms were as follows: 
(inflammatory bowel disease OR IBD OR ulcerative proctocolitis OR ulcerative colitis OR Crohn's disease) and (prostate cancer OR prostatic neoplasms OR prostatic cancer OR prostate neoplasms OR extraintestinal cancer OR extracolonic cancer). The cited references of reviews and the retrieved papers were also screened for additional relevant studies.

\section{Study selection}

All included studies had to fulfill the following criteria: (1) use a cohort study design; (2) assess the association between IBD and risk of PCa; (3) provide risk estimates with corresponding $95 \%$ confidence interval (Cls), or sufficient information to calculate these. When multiple reports from overlapping study cohort were published, the most recent or complete one was considered.

\section{Data extraction}

Two trained investigators independently reviewed and collected the following characteristics from each included study: author, publication year, country, study design, study population, study period, mean/median follow-up time, number of IBD case, and subtype of IBD. Newcastle-Ottawa scale (NOS) was applied to evaluate the quality of the cohort studies [24]. Studies given NOS scores of $0-4,5-6$, and 7-9 were considered be of low, moderate, and high quality, respectively. The conflicting opinions were settled by discussing with the third reviewer.

\section{Statistical analysis}

We used the relative risk (RR) estimates with $95 \% \mathrm{Cl}$ to assess the association between IBD and risk of PCa. Standardized incidence ratios and hazard ratios were directly considered as RRs because PCa incidence was low. In certain studies, risk estimates were stratified based on subtype of IBD, and risk of the IBD group was not provided. We calculated the summary RRs by the inverse-variance method. Interstudy heterogeneity was examined by Cochrane's $Q$ and the $R^{2}$ statistic. $P>0.1$ and $R^{2}<50 \%$ indicated an acceptable level of heterogeneity, and a fixed-effects model was applied. $P<0.1$ suggested the variation was significantly heterogeneous and a random-effects model was adopted. To explore the sources of heterogeneity across studies, subgroup analyses were conducted according to IBD type, region, and publication date. In addition, sensitivity analyses were performed to test the robustness of the pooled results by sequentially removing each single study. Publication bias was carried out by Begg's funnel plot, further confirmed by Begg's adjusted rank correlation test and Egger's linear regression test [25, 26]. $p<$ 0.05 was considered statistically significant. Meta-analyses were done with STATA 12.0 software (College Station, TX).

\section{Results}




\section{Literature search}

The search strategy generated 1249 potentially relevant articles from electronic databases (Fig 1). We excluded 1217 studies after screening the title and abstract of each study. We further assessed the remaining 32 potentially eligible articles by full-text review. Finally, a total of 14 articles [7, 9-21], comprising 13 cohort studies, fulfilled the predefined inclusion criteria and were eventually selected.

\section{Study characteristics}

The main characteristics of the 13 included studies are summarized in Table 1. These studies were published between 1991 and 2019. Among them, 11 retrospective population-based cohort studies [7, 10, 11, 13-21], 1 retrospective hospital-based cohort study [9] and 1 prospective population-based cohort study [12]. Eight studies were conducted in Europe, three in North America and two in Asia. The numbers of IBD patients in each study ranged from 756 to 49394 , comprising 210160 IBD cases. The quality scores in all cohorts were 6 or more stars.

\section{Meta-analysis results}

The pooled analyses demonstrated that IBD significantly elevated risk of prostate cancer (RR $1.36,95 \% \mathrm{Cl}$ 1.11-1.67, $Q=117.37, P_{\text {heterogeneity }}=0.003, P^{2}=89.8 \%$ ) (Fig 2). Further subgroup analysis was carried out according to IBD type, study location, and publication date (Table 2). The results showed the increased risk of PCa was associated with UC (RR 1.30, 95\% Cl 1.09-1.55) but not CD (RR 1.09, 95\% Cl 0.90-1.34). IBD showed a statistically significant association with PCa risk among studies conducted in Asia (RR $3.02,95 \% \mathrm{Cl} 2.24-4.48$ ), however, no significant association was noted among studies conducted in Europe (RR 1.16, 95\% Cl 0.99-1.36) or Northern America (RR 1.56, 95\% Cl 0.55-4.42). In addition, we conducted subgroup analyses by publication year, using 2010 as the cutoff. We observed a positive association between IBD and risk of PCa in the cohort studies published after 2010 (RR $1.53,95 \% \mathrm{Cl}$ 1.14-2.07) but no significant association was noted among studies published before 2010 (RR 1.11, $95 \% \mathrm{Cl} 0.91-1.35)$.

\section{Sensitivity analysis}

In the sensitivity analysis, none of the studies considerably influenced the pooled estimates were observed in the meta-analysis, suggesting that our conclusion was robust.

\section{Publication bias}

Begg's funnel plot (Fig 3), together with the Begg's and Egger's test $\left(P_{\text {Begg's }}=0.669, P_{\text {Egger's }}=0.362\right)$, did not suggest any evidence of publication bias. 


\section{Discussion}

Previously, a meta-analysis conducted by Pedersen et al. included eight population-based cohort studies and found that patients suffering from UC were at elevated risk of liver-biliary cancer, and that patients suffering from $C D$ were at elevated risk of upper gastrointestinal tract, lung, urinary tract and skin cancer. However, their pooled results did not demonstrate a relationship between the history of IBD and the risk of PCa, neither among patients with UC nor with CD [6]. In our present meta-analysis, thirteen cohort studies comprising 210160 patients with IBD were included. Our results showed that IBD significantly increased risk of PCa. Even though a greater heterogeneity was observed in studies, sensitivity analysis showed the robustness of our conclusion.

In subgroup analyses, we firstly explored the association between IBD and PCa by the subtype of IBD. We found that UC significantly increased risk of PCa, while CD was not. The discrepancy is possibly due to the different areas affected in the gastrointestinal tract between UC and CD. CD may involve any area of the gastrointestinal tract, from mouth to anus, even though most of lesions start in the end of the small bowel (the ileum) and the beginning of the colon. UC, however, is limited to the colon and the rectum. Patients with UC usually present with diarrhea mixed with blood and are more likely to have digital rectal examinations, which may promote the finding of PCa. We also performed subgroup analyses by geographic location to explore the influence of different areas. The results showed that IBD significantly increased risk of PCa among studies conducted in Asia, but no significant relationship was noted among studies conducted in Europe or Northern America. This finding indicated that geographic distribution may play roles in the carcinogenesis of PCa for IBD patients.

In recent years, the immunosuppressant drugs have been widely administered to IBD patients. But several studies revealed that long-term immunosuppressive use may contribute to the development of cancer [27, 28]. Due to the large span of study period and absence of sufficient information, we failed to explore the influence of medications use on our conclusion. Therefore, we conducted subgroup analyses by publication year. We observed a positive association between IBD and risk of PCa in the cohort studies published after 2010 but the association was insignificant among studies published before 2010 . The discrepancy may somewhat due to the medications use mentioned above. Therefore, further studies on this issue are needed.

Potential biological mechanisms linked IBD to PCa remain unclear. But several reasonable explanations have been proposed. IBD exerts a local or systemic inflammatory environment, which cause chronic prostatic inflammation and contribute to prostate carcinogenesis [29]. As the longer duration of the chronic inflammation persists, there is an increasing risk of cancer development [30]. Furthermore, immunosuppression therapy for IBD will mitigate the autoimmune activity and cause lymphoproliferative disorder [31]. Immunosuppressant medications also may decrease tumor surveillance, impaired ability to fight chronic infections, and direct DNA damage, which is related to the development of extraintestinal cancer $[27,28]$. Additionally, patients suffering from IBD trend to seek for help from healthcare providers frequently and are more likely to have prostate-specific antigen and digital rectal examinations, which 
may promote the finding of PCa [32]. Last but not least, IBD has common susceptibility alleles with PCa, such as folate hydrolase 1 (FOLH1) / prostate-specific membrane antigen (PSMA) $[9,33,34]$.

There are several limitations needed to be concern in our meta-analysis. First, the heterogeneity was significant, although we used a random effect model and conducted subgroup analysis to lessen them partially. Secondly, most studies were retrospective cohort design, which could bring recall bias. Thirdly, although most of included studies had performed adjustment for some confounders, we could not rule out the possibility that other unidentified or unmeasured factors, such as the use of medications, could affect the association. Finally, we fail to clarify the influence of IBD disease duration on risk of PCa without enough data.

\section{Conclusions}

Our analysis suggests the risk of PCa is significantly increased among IBD patients, particularly among UC males. Physicians should be cognizant of the relationship and suggest a regular PCa screening when treating men with IBD. However, the possibility that the association may be influenced by bias or confounding variables. More prospective cohort studies are required to confirm our findings. The mechanisms responsible for this association also warrant to be clarified.

\section{Abbreviations}

PCa: prostate cancer; IBD: inflammatory bowel disease; CD: Crohn's disease; UC: ulcerative colitis; Cl: confidence interval; NOS: Newcastle-Ottawa scale; RR: relative risk; FOLH1: folate hydrolase 1; PSMA: prostate-specific membrane antigen.

\section{Declarations}

Acknowledgments『Not applicable.

\section{Funding $\mathbb{N}$ None.}

Authors' contributions: WQL: project development, data analysis, manuscript writing/editing. $\mathrm{HXH}$ and FJL: data collection, data analysis. LHC: data collection, manuscript writing/editing. All authors read and approved the final manuscript.

Ethical approval and consent to participate $₫ A$ s this meta-analysis was based on published studies $₫$ we did not apply for the approval of institutional review board.

Availability of data and materials: All data during this study were presented within the manuscript.

Consent for publication®Not applicable.

Conflict of interest: The authors declare that they have no conflict of interest. 


\section{References}

1Bray F, Ferlay J, Soerjomataram I, Siegel RL, Torre LA, Jemal A: Global cancer statistics 2018: Globocan estimates of incidence and mortality worldwide for 36 cancers in 185 countries. CA: a cancer journal for clinicians 2018;68:394-424.

2Jiang J, Li J, Yunxia Z, Zhu H, Liu J, Pumill C: The role of prostatitis in prostate cancer: Meta-analysis. PloS one 2013;8:e85179.

3Platz EA, De Marzo AM: Epidemiology of inflammation and prostate cancer. The Journal of urology 2004;171:S36-40.

4Dennis LK, Lynch CF, Torner JC: Epidemiologic association between prostatitis and prostate cancer. Urology 2002;60:78-83.

5Sartor RB: Mechanisms of disease: Pathogenesis of crohn's disease and ulcerative colitis. Nature clinical practice Gastroenterology \& hepatology 2006;3:390-407.

6Pedersen N, Duricova D, Elkjaer M, Gamborg M, Munkholm P, Jess T: Risk of extra-intestinal cancer in inflammatory bowel disease: Meta-analysis of population-based cohort studies. The American journal of gastroenterology 2010;105:1480-1487.

7Loo SY, Vutcovici M, Bitton A, Lakatos PL, Azoulay L, Suissa S, Brassard P: Risk of malignant cancers in inflammatory bowel disease. Journal of Crohn's \& colitis 2019

8Mosher CA, Brown GR, Weideman RA, Crook TW, Cipher DJ, Spechler SJ, Feagins LA: Incidence of colorectal cancer and extracolonic cancers in veteran patients with inflammatory bowel disease. Inflammatory bowel diseases 2018;24:617-623.

9Burns JA, Weiner AB, Catalona WJ, Li EV, Schaeffer EM, Hanauer SB, Strong S, Burns J, Hussain MHA, Kundu SD: Inflammatory bowel disease and the risk of prostate cancer. European urology 2018

10So J, Tang W, Leung WK, Li M, Lo FH, Wong MTL, Sze ASF, Leung CM, Tsang SWC, Shan EHS, Chan KH, Lam BCY, Hui AJ, Chow WH, Lam TY, Lam V, Lee TW, Lo HHH, Tang CM, Wong CL, Wu JCY, Chan FKL, Sung JJY, Harbord M, Ng SC: Cancer risk in 2621 chinese patients with inflammatory bowel disease: A population-based cohort study. Inflammatory bowel diseases 2017;23:2061-2068.

11 Jung YS, Han M, Park S, Kim WH, Cheon JH: Cancer risk in the early stages of inflammatory bowel disease in korean patients: A nationwide population-based study. Journal of Crohn's \& colitis 2017;11:954-962.

12 Hovde $\emptyset$, Hoivik ML, Henriksen M, Solberg IC, Smastuen MC, Moum BA: Malignancies in patients with inflammatory bowel disease: Results from 20 years of follow-up in the ibsen study. Journal of Crohn's \& colitis 2017;11:571-577. 
13Wilson JC, Furlano RI, Jick SS, Meier CR: A population-based study examining the risk of malignancy in patients diagnosed with inflammatory bowel disease. Journal of gastroenterology 2016;51:1050-1062.

14Kappelman MD, Farkas DK, Long MD, Erichsen R, Sandler RS, Sorensen HT, Baron JA: Risk of cancer in patients with inflammatory bowel diseases: A nationwide population-based cohort study with 30 years of follow-up evaluation. Clinical gastroenterology and hepatology: the official clinical practice journal of the American Gastroenterological Association 2014;12:265-273.e261.

15Jussila A, Virta LJ, Pukkala E, Farkkila MA: Malignancies in patients with inflammatory bowel disease: A nationwide register study in finland. Scandinavian journal of gastroenterology 2013;48:1405-1413.

16Jess T, Horvath-Puho E, Fallingborg J, Rasmussen HH, Jacobsen BA: Cancer risk in inflammatory bowel disease according to patient phenotype and treatment: A danish population-based cohort study. The American journal of gastroenterology 2013;108:1869-1876.

17Hemminki K, Li X, Sundquist J, Sundquist K: Cancer risks in crohn disease patients. Annals of oncology: official journal of the European Society for Medical Oncology 2009;20:574-580.

18Hemminki K, Li X, Sundquist J, Sundquist K: Cancer risks in ulcerative colitis patients. International journal of cancer 2008;123:1417-1421.

19Bernstein CN, Blanchard JF, Kliewer E, Wajda A: Cancer risk in patients with inflammatory bowel disease: A population-based study. Cancer 2001;91:854-862.

20Karlen P, Lofberg R, Brostrom O, Leijonmarck CE, Hellers G, Persson PG: Increased risk of cancer in ulcerative colitis: A population-based cohort study. The American journal of gastroenterology 1999;94:1047-1052.

21Ekbom A, Helmick C, Zack M, Adami HO: Extracolonic malignancies in inflammatory bowel disease. Cancer 1991;67:2015-2019.

22Moher D, Liberati A, Tetzlaff J, Altman DG, Group P: Preferred reporting items for systematic reviews and meta-analyses: The prisma statement. International journal of surgery 2010;8:336-341.

23Stroup DF, Berlin JA, Morton SC, Olkin I, Williamson GD, Rennie D, Moher D, Becker BJ, Sipe TA, Thacker SB: Meta-analysis of observational studies in epidemiology: A proposal for reporting. Meta-analysis of observational studies in epidemiology (moose) group. Jama 2000;283:2008-2012.

24Stang A: Critical evaluation of the newcastle-ottawa scale for the assessment of the quality of nonrandomized studies in meta-analyses. European journal of epidemiology 2010;25:603-605.

25Begg CB, Mazumdar M: Operating characteristics of a rank correlation test for publication bias. Biometrics 1994;50:1088-1101. 
26Egger M, Davey Smith G, Schneider M, Minder C: Bias in meta-analysis detected by a simple, graphical test. Bmj 1997;315:629-634.

27Yadav S, Singh S, Harmsen WS, Edakkanambeth Varayil J, Tremaine WJ, Loftus EV, Jr.: Effect of medications on risk of cancer in patients with inflammatory bowel diseases: A population-based cohort study from olmsted county, minnesota. Mayo Clinic proceedings 2015;90:738-746.

28Biancone L, Onali S, Petruzziello C, Calabrese E, Pallone F: Cancer and immunomodulators in inflammatory bowel diseases. Inflammatory bowel diseases 2015;21:674-698.

29Nakai Y, Nonomura N: Inflammation and prostate carcinogenesis. International journal of urology: official journal of the Japanese Urological Association 2013;20:150-160.

30Axelrad JE, Lichtiger S, Yajnik V: Inflammatory bowel disease and cancer: The role of inflammation, immunosuppression, and cancer treatment. World journal of gastroenterology 2016;22:4794-4801.

31Subramaniam K, D'Rozario J, Pavli P: Lymphoma and other lymphoproliferative disorders in inflammatory bowel disease: A review. Journal of gastroenterology and hepatology 2013;28:24-30.

32Kappelman MD, Porter CQ, Galanko JA, Rifas-Shiman SL, Ollendorf DA, Sandler RS, Finkelstein JA: Utilization of healthcare resources by u.S. Children and adults with inflammatory bowel disease. Inflammatory bowel diseases 2011;17:62-68.

33Rais R, Jiang W, Zhai H, Wozniak KM, Stathis M, Hollinger KR, Thomas AG, Rojas C, Vornov JJ, Marohn $\mathrm{M}, \mathrm{Li}$ X, Slusher BS: Folh1/gcpii is elevated in ibd patients, and its inhibition ameliorates murine ibd abnormalities. JCl insight 2016;1

34Ghosh A, Heston WD: Tumor target prostate specific membrane antigen (psma) and its regulation in prostate cancer. Journal of cellular biochemistry 2004;91:528-539.

\section{Tables}

Table 1. Characteristics of the cohort studies included in the meta-analysis 


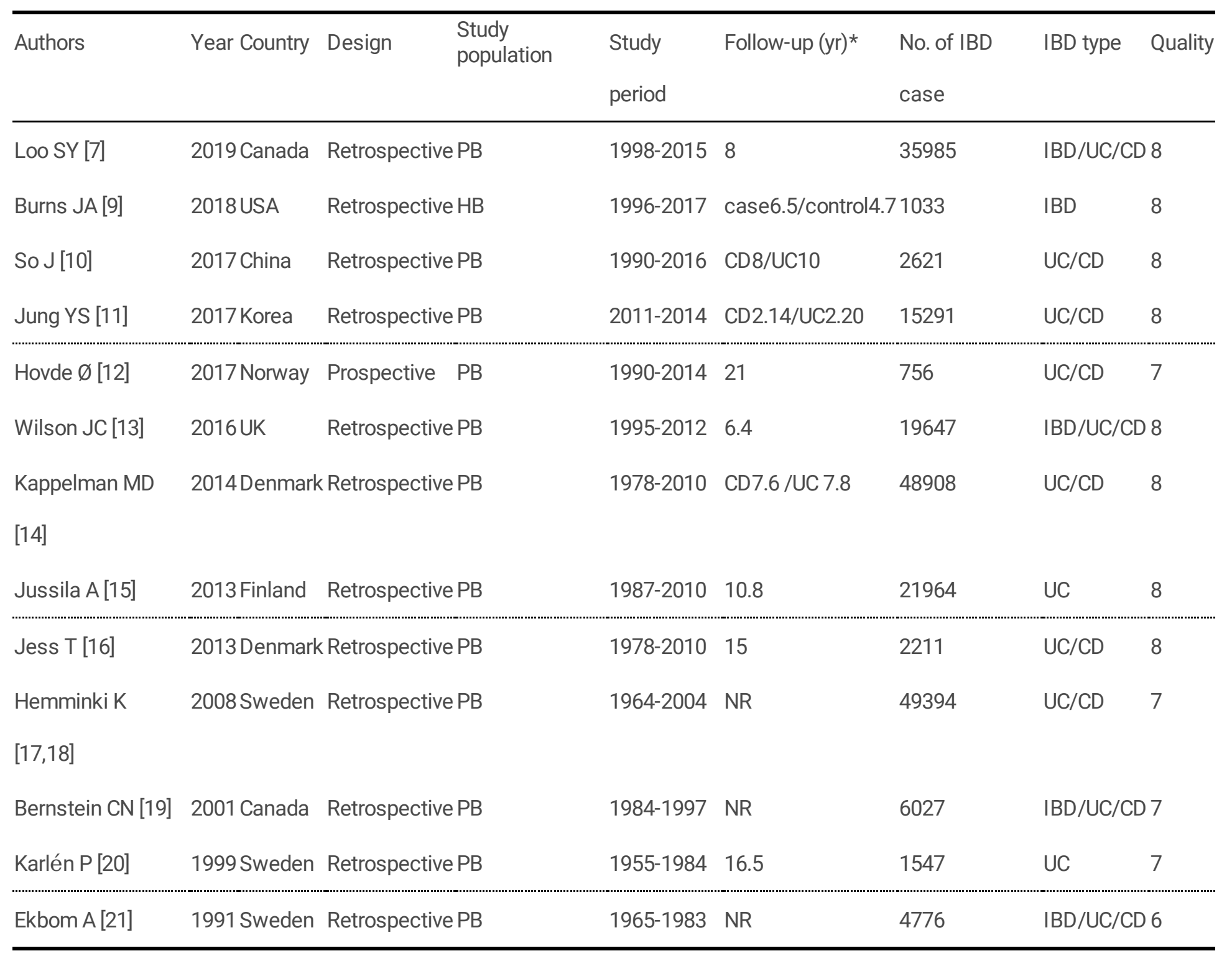

Abbreviations: PB, population-based; HB, hospital-based; CD, Crohn's disease; UC, ulcerative colitis; IBD, inflammatory bowel disease; NR, not reported.

*Mean or mean duration of follow-up.

Table 2. Overall and subgroup analyses of the association between inflammatory bowel disease and the risk of prostate cancer 
Study heterogeneity

\begin{tabular}{|c|c|c|c|c|c|c|}
\hline \multicolumn{2}{|l|}{ Subgroup } & \multicolumn{2}{|c|}{ Studies, n OR (95\% Cl) } & $Q$ & $P$ & \multirow{2}{*}{$\frac{R_{, \%}}{89.8}$} \\
\hline Overall & & 13 & $1.36(1.11-1.6$ & 7) 117.37 & 0.003 & \\
\hline \multirow[t]{2}{*}{ IBD type } & UC & 11 & $1.30(1.09-1.5$ & 5) 32.98 & 0.004 & 69.7 \\
\hline & $\mathrm{CD}$ & 4 & $1.09(0.90-1.3$ & 4) 28.47 & 0.377 & 68.4 \\
\hline \multirow[t]{3}{*}{ Geographic regio } & Europe & 8 & $1.16(0.99-1.3$ & 32.40 & 0.071 & 78.4 \\
\hline & North America & & $1.56(0.55-4.4$ & 2) 62.13 & 0.404 & 96.8 \\
\hline & Asia & 2 & $3.02(2.24-4.4$ & 3) 0.48 & $\square 0.001$ & 0 \\
\hline \multirow[t]{2}{*}{ Publication year } & $\geq 2010$ & 9 & $1.53(1.14-2.0$ & 7) 111.20 & 0.005 & 92.8 \\
\hline & $<2010$ & 4 & $1.11(0.91-1.3$ & 5) 4.41 & 0.303 & 31.9 \\
\hline
\end{tabular}

Figures 
Relevant studies identified from

Embase $(n=807)$, Pubmed $(n=409)$,

Cochrane Library $(n=33)$

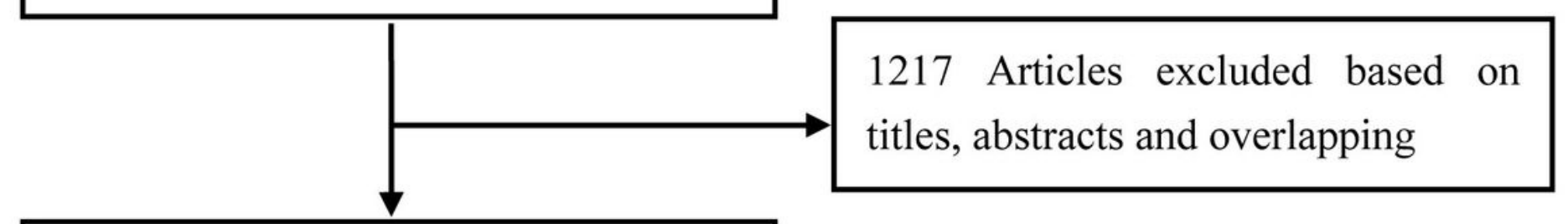

Full-text articles reviewed for further evaluation $(n=32)$

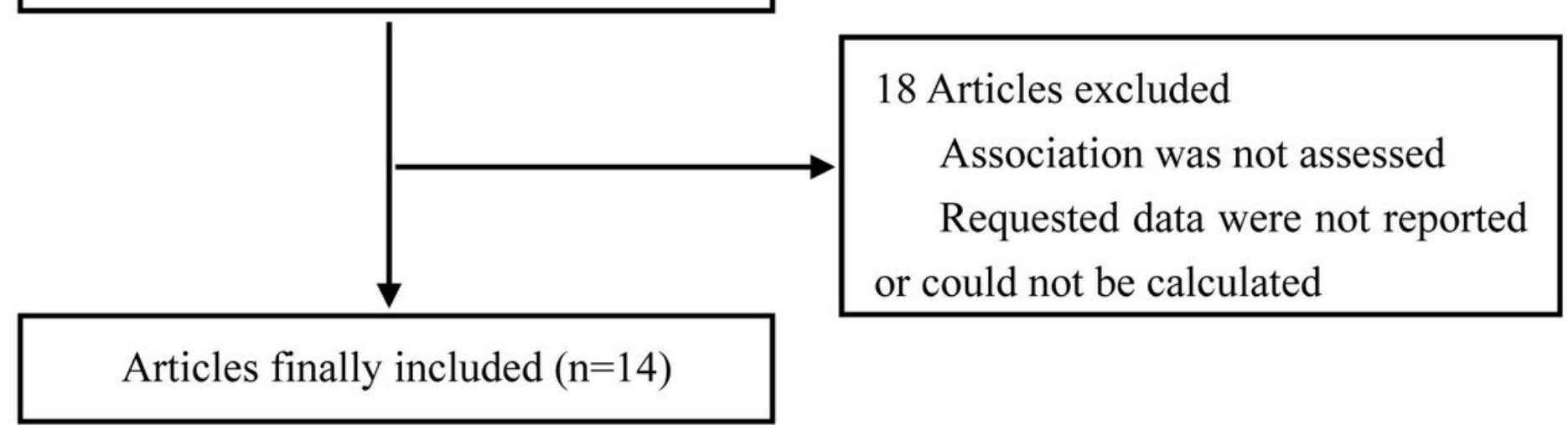

Figure 1

Flowchart of literature search and study selection 
Study

ID
$\%$

$\mathrm{RR}(95 \% \mathrm{Cl}) \quad$ Weight

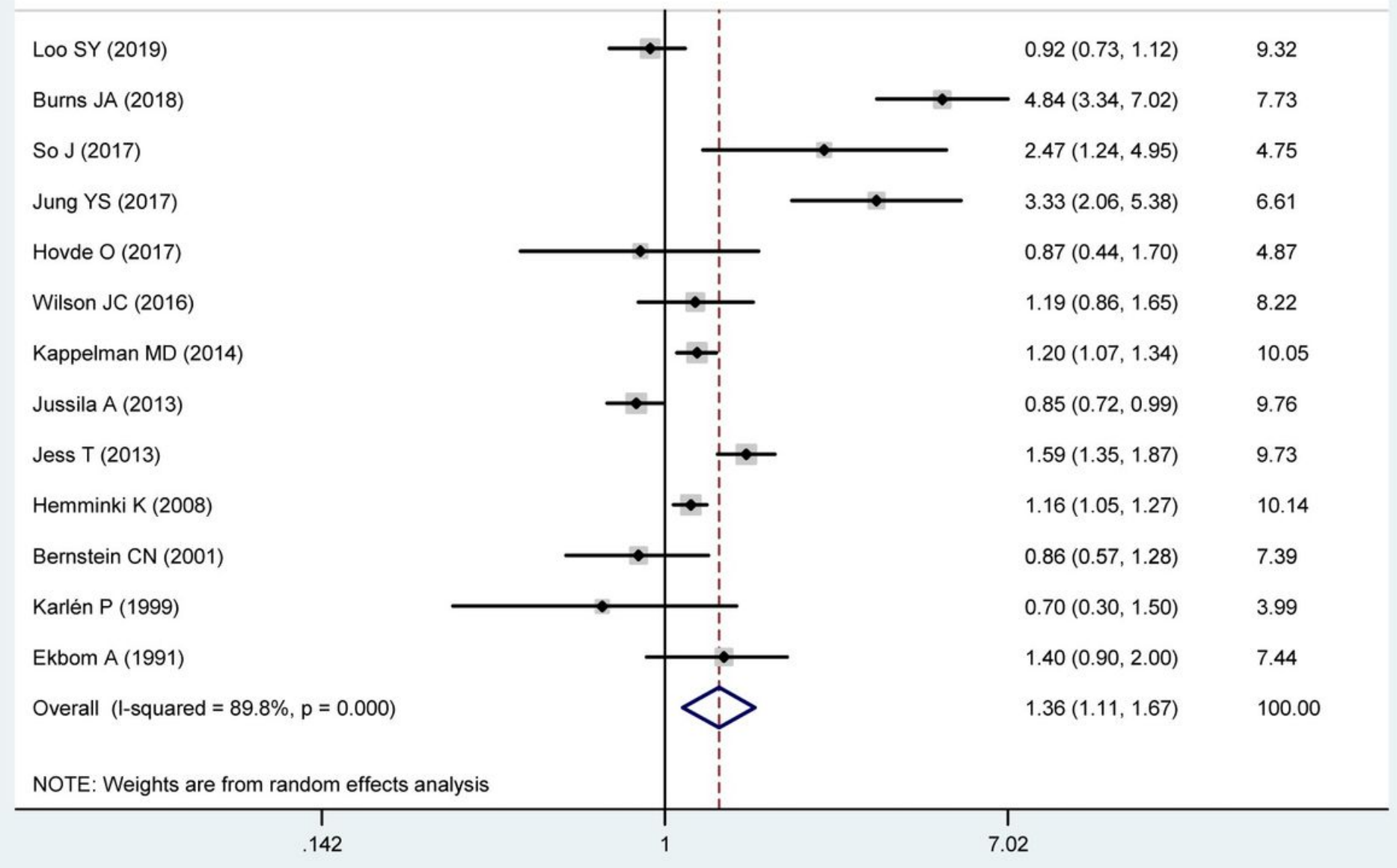

\section{Figure 2}

Forest plot for inflammatory bowel disease and risk of prostate cancer 
Begg's funnel plot with pseudo $95 \%$ confidence limits

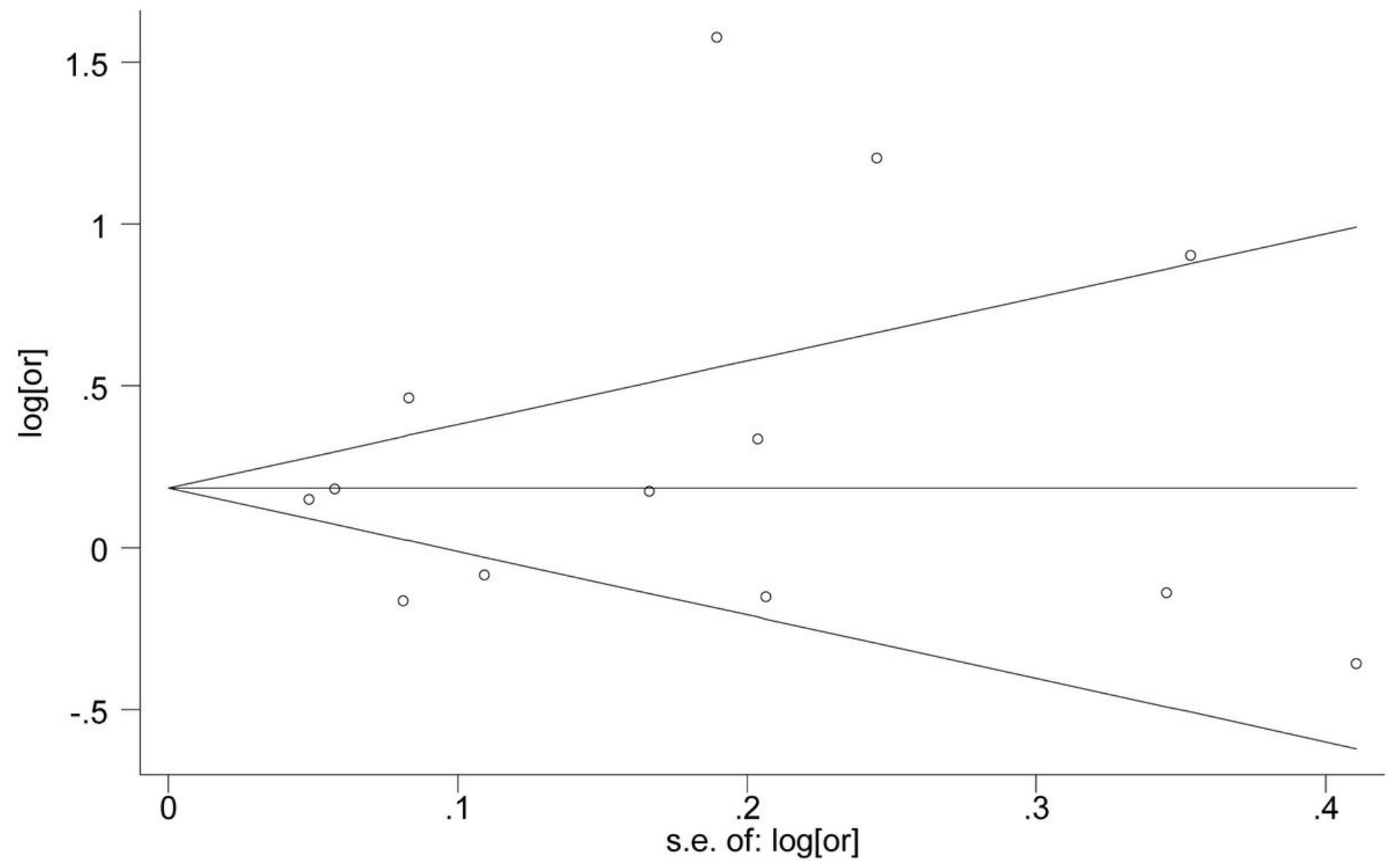

Figure 3

Begg's funnel plot of inflammatory bowel disease and prostate cancer risk

\section{Supplementary Files}

This is a list of supplementary files associated with this preprint. Click to download.

- Additionalfile1DataS1PRISMA2009checklist.doc 